\title{
LABORATORIO VIRTUAL PARA LA ENSEÑANZA DE FILTROS ELÉCTRICOS
}

Por:

José Ricardo Bermúdez Santaella' ${ }^{1}$, Jaime Ramírez Arbeláez²

\section{SUMMARY}

This article describes to the design and implementation of a virtual laboratory, for the education of electrical filters. The virtual laboratory is implemented in an atmosphere of windows for which the student or teacher does not require a previous knowledge to handle the program. The virtual laboratory simulates: a) A generator of functions, which can produce a signal of sinusoidal type, square, triangular and tooth of mountain range. For each signal it is possible to be selected the amplitude, frequency and the cycle to it of work (square signal). b) Generates a filter of Butterworth or Chebychev. c) It allows to the visualization of the in-out signals and exit of the filter, as much in the dominion of the time like in frequency.

\section{RESUMEN}

Este artículo describe el diseño e implementación de un laboratorio virtual, para la enseñanza de filtros eléctricos. El laboratorio virtual está implementado en un ambiente de ventanas para el cual el estudiante o maestro no requiere un conocimiento previo para manejar el programa. El laboratorio virtual simula: a) Un generador de funciones, el cual puede producir una señal de tipo sinusoidal, cuadrada, triangular y diente de sierra. Para cada señal se le puede seleccionar la amplitud, frecuencia y el ciclo de trabajo (señal cuadrada). b) Genera un filtro de Butterworth o Chebychev. c) Permite la visualización de las señales de entrada y salida del filtro, tanto en el dominio del tiempo como en frecuencia.

Palabras claves: LabView, Butterworth, chebychev, Interfaz, tiempo, frecuencia.

\section{INTRODUCCIÓN}

Tradicionalmente, la enseñanza teórica de filtros eléctricos se lleva a cabo mediante clases magistrales donde los conceptos teóricos no pueden ser verificados con trabajos de laboratorios y además se hace difícil ilustrar gráficamente el efecto en el tiempo y en la frecuencia, que producen los filtros en el tratamiento de las señales eléctricas . También, teniendo en cuenta que los filtros eléctricos son parte de los acondicionadores de señal de casi todos los sensores eléctricos y que en general la gran mayoría de equipos electrónicos posee al menos un filtro eléctrico, es importante que los estudiantes comprendan claramente el concepto de filtro eléctrico. En el plan de estudios de la Universidad de los Andes (Mérida, Venezuela) se ha planteado la enseñanza de los filtros eléctricos como un curso teórico-práctico que incluye una parte teórica reforzada con un laboratorio virtual de filtros y un grupo de prácticas de laboratorio, donde se diseña y experimenta con diferentes tipos de filtros pasivos y activos.

En este artículo se presenta el laboratorio virtual como una herramienta pedagógica que permite al estudiante visualizar los efectos en el tiempo y en la frecuencia, que produce los filtros eléctricos en el tratamiento de las señales.

\section{LABORATORIO VIRTUAL DE FILTROS ELECTRICOS}

El desarrollo de la instrumentación virtual [1] ha seguido muy de cerca el avance de la industria de los computadores y en la medida en que los computadores personales se han hecho más asequibles, se ha masificado su uso como una herramienta pedagógica.

Un instrumento virtual, en principio, es un instrumento basado en un computador y manejado

\footnotetext{
'Universidad Francisco de Paula Santander, grupo de investigación de desrrollo en procesos industriales "GIDPI" jrbermud@bari.ufps.edu.co

2 Universidad de los Andes, postgrado en automatización e instrumentación, jramirez@ula.ve
} 
por un programa usado para pruebas, mediciones y control de procesos.

El laboratorio virtual de filtros eléctricos consiste en el desarrollo de un instrumento virtual diseñado, usando el lenguaje de programación gráfico ( $G$ ) del LabView[2], que permite ver en forma inmediata las señales de entrada y salida y el espectro de frecuencias de datos para diferentes tipos de filtros eléctricos.

\section{INTERFAZ DEL USUARIO}

La figura 1. muestra la pantalla principal del laboratorio virtual.

La parte izquierda e inferior de la pantalla se usa para introducir los datos que ajustan los valores de la señal producida por el generador y permiten la selección del filtro que se quiere analizar.

En la parte derecha y central de la pantalla se muestra la representación gráfica de las señales en el dominio del tiempo y en el dominio de la frecuencia. La señal generada y el tipo de filtro se pueden modificarse.

Figura 1. Panel principal.

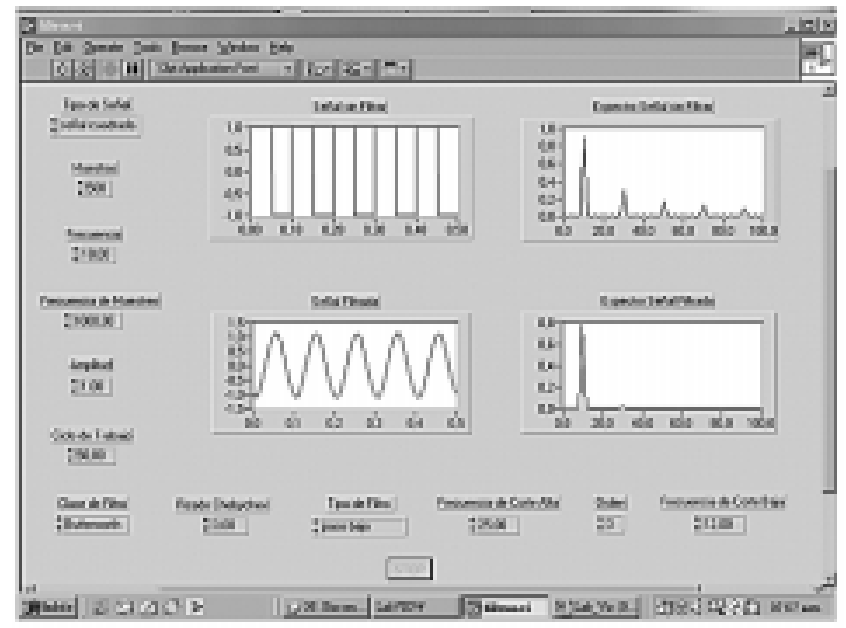

3.1 Conformación del panel de control

- Tipo de señal: Cuadrada, Sinusoidal, Triangular y Diente de sierra, ver figura 2.
Figura 2. Cuadro de control

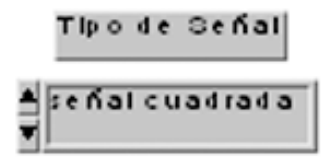

Se utiliza un control "List \& Ring ( Text Ring )", controles similares al anterior se utilizan para seleccionar la clase y tipo de filtro.

- Clase de filtro:

Butterworth y Chebychev.

- Tipo de filtro:

Pasa bajo, Paso alto, Paso banda y Banda eliminada.

Para introducir el resto de información necesaria se usan controles digítales numéricos como el siguiente, ver figura 3 :

Figura 3. Control digital

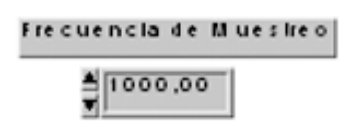

Los otros controles que se presentan en el panel frontal son:

- Muestras.

- Frecuencia de la señal.

- Frecuencia de muestreo.

- Amplitud de la señal.

- Ciclo de trabajo de la señal cuadrada.

- Rizado del filtro Chebycher en dB.

- Frecuencia de corte alta.

- Frecuencia de corte baja.

- Orden del filtro.

Para mostrar las señales en forma gráfica utilizamos el control "Graph "y seleccionamos "wareform graph" como se muestra, ver figura 4 : 
Figura 4. Control gráfico.

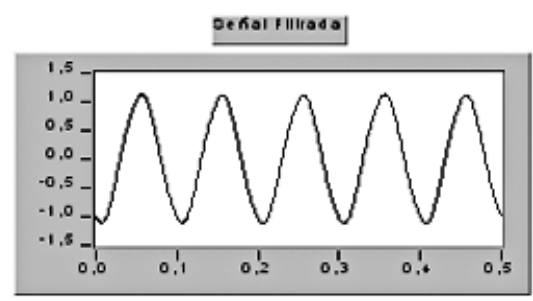

3.2 Conformación del laboratorio virtual con los siguientes instrumentos, ver figura 5 :

Figura 5. Estructura del laboratorio virtual.

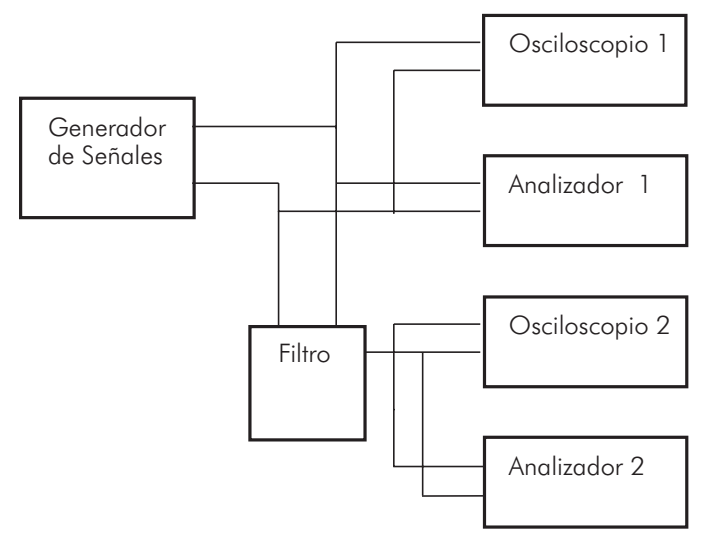

En el generador de señales se puede fijar: Amplitud, Frecuencia, Número de muestra (n), Frecuencia de muestreo (f), Tipo de señal, Ciclo de trabajo para la onda cuadrada.

La señal que se obtiene del generador se aplica a un osciloscopio para ver la forma de onda en función del tiempo. También se conecta a un analizador de espectro para ver el espectro de frecuencia de la señal (valor RMS de los armónicos vs frecuencia ). Luego la señal se adapta a un filtro donde se puede seleccionar: la clase del filtro, el tipo de filtro, frecuencia de corte alta, frecuencia de corte baja, orden del filtro y el rizado en $\mathrm{dB}$.

En este laboratorio virtual se puede obtener también la señal filtrada y el espectro de la señal filtrada. Aquí podemos ver en tiempo real el efecto de cambiar tipos de filtros, orden del filtro, frecuencias de corte, sobre la señal aplicada al filtro.
3.2.1 Conformación del panel de diagrama, ver figura 6.

Figura 6. Panel de diagrama.

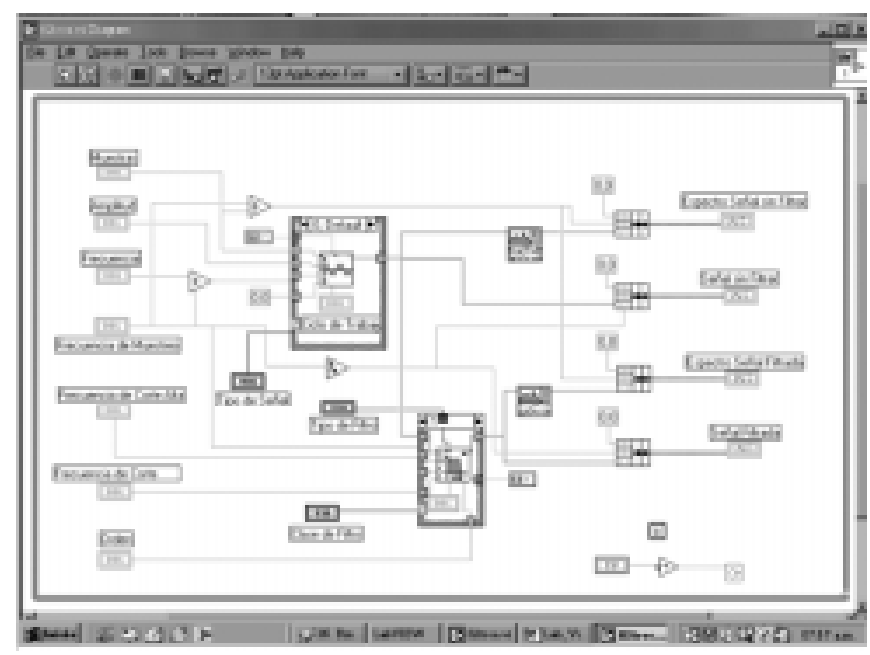

Si analizamos el panel de diagrama, vemos que para obtener el generador de funciones se usan los siguientes instrumentos virtuales:

"funciones \Análisis \Signal generation", ver figura 7 .

$y[i]=a m p * \sin ($ phase $[i])$, for $i=0,1,2, \ldots, n-1$ (1)

donde:

Figura 7. Estructura de conexión interna.

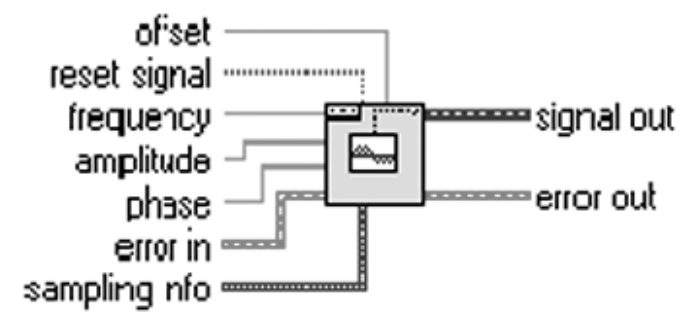

Fase $[I]=$ Fase de entrada $+f^{*} 360 * I$

La frecuencia f, la llamaremos frecuencia normalizada y la podemos obtener de dos formas distintas: 
- $\mathrm{fe} / \mathrm{n}=$ frecuencia normalizada (ciclos/muestra).

En este caso, fe, se interpreta como el número de ciclos que se quiere que aparezcan en pantalla.

- $\mathrm{fe} / \mathrm{fs}_{\mathrm{s}}=$ frecuencia normalizada (ciclos/muestra)

La forma anterior se utiliza en este laboratorio. El máximo número de muestras depende de la memoria del sistema, teóricamente, limitado a $2^{31}-1$. Sin embargo el procesamiento digital de la señal y el tiempo de procesador deben tenerse en cuenta.

En este trabajo se fija la fase de entrada o cero grado a " verdadero "lo cual hace que en cada nueva corrida la fase de entrada se coloca siempre en cero.

El tiempo mostrado de la señal está dado por $1 / \mathrm{fs}^{*} 100$, en cual se ajusta en el "Bundle" ver figura 8.

Figura 8. Bundle.

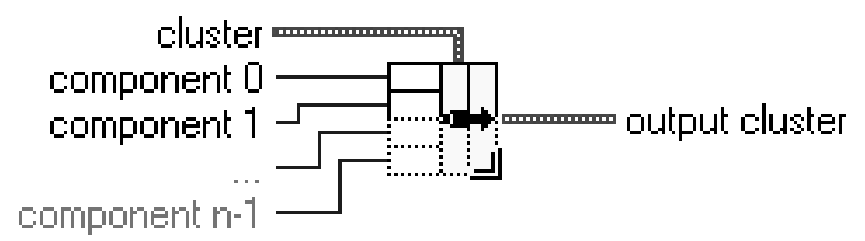

En este laboratorio virtual se presenta la forma de onda entre 0 a 0,1 seg.

Para el analizador de espectro se tiene $f_{s} / n,=10$, si queremos disminuir el paso podemos aumentar el número de muestras.

Para seleccionar el tipo de señal se usa un " Case Structure "ver figura 9, que está conectado a un control " List \& Ring (Text Ring)" que permite seleccionar el tipo de señal en cada Case, se incluye el generador de onda correspondiente.

Figura 9. Estructura case.

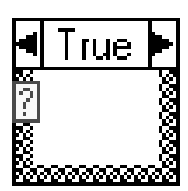

Solo hay que tener en cuenta que el "case" de onda cuadrada tiene un control adicional correspondiente al control del ciclo de trabajo de la onda (\% ). También se usa un "Case Structure" para seleccionar la clase de filtro.

Para presentar las ondas y sus espectros se usan las funciones "Cluster"/ "Bundel". Emsambla todas las componentes individuales en un cluster simple, este ensamble permite controlar el eje $x$ de las presentaciones.

Otras funciones utilizadas en este laboratorio virtual son:

"Functions \Análisis \Filtres \(Butterworth o Chebycher)".

Butterworth filter.vi: ver figura 10

Figura 10. Filtro Butterworth.

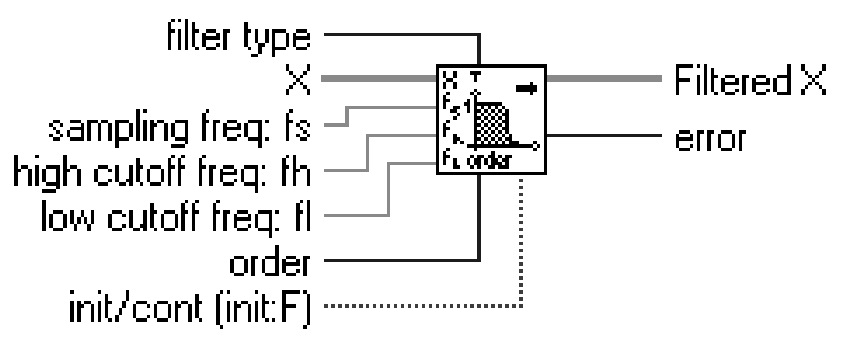

La frecuencia de muestreo ts debe ser mayor o igual a cero, si es menor que cero da como resultado un arreglo vacio y retorna un error tn es ignorada cuando el filtro es paso-bajo o paso-alto, la frecuencia de corte baja tiene que cumplir con el criterio de Nyquist $0<=$ te $<=0.5$ ts.

Cuando es falso los estados internos son inicializados a cero. Cuando es verdadero los estados del filtro son inicializados a los estados de la corrida previa.

Para los filtros de Butterworth siempre se usa un am $=3$ $\mathrm{dB}$, los filtros de Butterworth no siempre proveen una buena aproximación a las respuestas de los filtros ideales debido a la pequeña pendiente en la zona de transición. 
Chebychev filter.vi: ver figura 11

Figura 11. Filtro Chebychev.

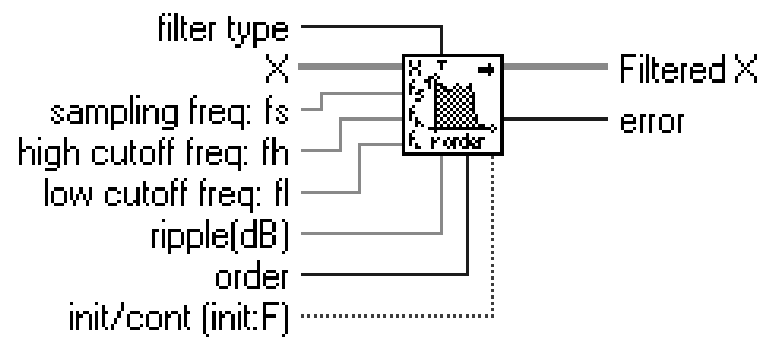

Los filtros de Chebychev minimizan el error pico en la banda de paso al permitir seleccionar el rizado en banda de paso. Presentan una mayor pendiente en la zona de transición que los filtros de Butterworth lo cual se manifiesta en filtros de más bajo orden lo cual produce errores menores y velocidades de ejecución mayores.

Los filtros de Chebychev requieren un control adicional correspondiente al rizado. En la demostración se usa el instrumento virtual "Amplitud and Phase Spectrum.vi" que se consigue con el panel de funciones:

"Funtions/Análisis/Measurement/Ampl. And Phase.vi", ver figura 12:

Antes de iniciar la descripción de este instrumento es conveniente tener presente los concepto sobre la transformada discreta de Fourier ( DFT ).

Figura 12. DTF.

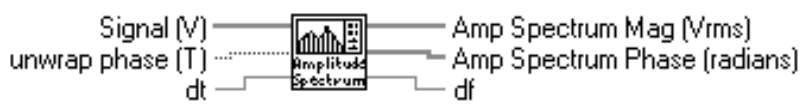

Amplitud and Phase Spectrum Analyzer.vi:

Computa la amplitud de espectro (Single sided) de una señal en el dominio del tiempo. La amplitud del espectro se computa como $\mathrm{FFT}(\mathrm{x}) / \mathrm{N}$ donde $\mathrm{N}$ es el número de puntos en el arreglo de la señal $x$. La amplitud del espectro se convierte en un espectro de magnitud y fase, controla el unwrapping de la fase del espectro Set a True para habilitar el unwrapping de la fase sobre el arreglo de salida del 2 Amp Spectrum Phase (radians), si se coloca en false el VI no ejecuta el unwrapping ( Phase $<=\mathrm{P} 1$ ) dt es el periodo de muestra de la señal en el tiempo, en segundos $d t=1$ / ts donde ts es la frecuencia de muestreo de la señal del tiempo.

En la forma que se crea conveniente. En la figura 6. se muestra la funcionalidad del instrumento virtual desarrollado.

\section{GENERADOR DE SEÑALES}

En esta sección se establecen las condiciones de las señales que pueden excitar el filtro:

- Señal sinusoidal

$$
x(t)=A \operatorname{sen}(2 \pi f t+\phi)
$$

A esta señal se le puede variar la amplitud ( A ) la frecuencia $(t)$.

\section{- Señal cuadrada}

A esta señal se le pueden variar la amplitud ( $A$ ), la frecuencia y el ciclo de trabajo $(\delta)$.

- Señal triangular y Señal diente de sierra

A esta señales se le puede variar la amplitud y la frecuencia.

Si calculamos la serie Fourier para cada una de estas señales obtenemos el respectivo espectro de frecuencias el cual se muestra a continuación.

El instrumento virtual diseñado nos permite observar la señal generada y su respectivo espectro de frecuencias. 


\section{FILTRO}

El filtro eléctrico que se usa para procesar la señal de entrada proveniente del generador de señales se puede elegir entre un filtro de Butterworth o un filtro de Chebychev.

Para cada uno de estos filtros se puede seleccionar el tipo de filtro:

a) Filtro pasa-bajo, paso-alto, banda y banda eliminada.

b) Frecuencia de corte.

c) Rizado en banda de paso para los filtros de Chebycher.

d) Orden del filtro.

La respuesta de los filtros se puede analizar a través de la respectiva función de transferencia. Mediante el instrumento virtual diseñado podemos observar el diagrama de Bode y la respuesta impulsiva del filtro aplicado, ver figura 13.

Figura 13. Diagrama de bode e implusiva

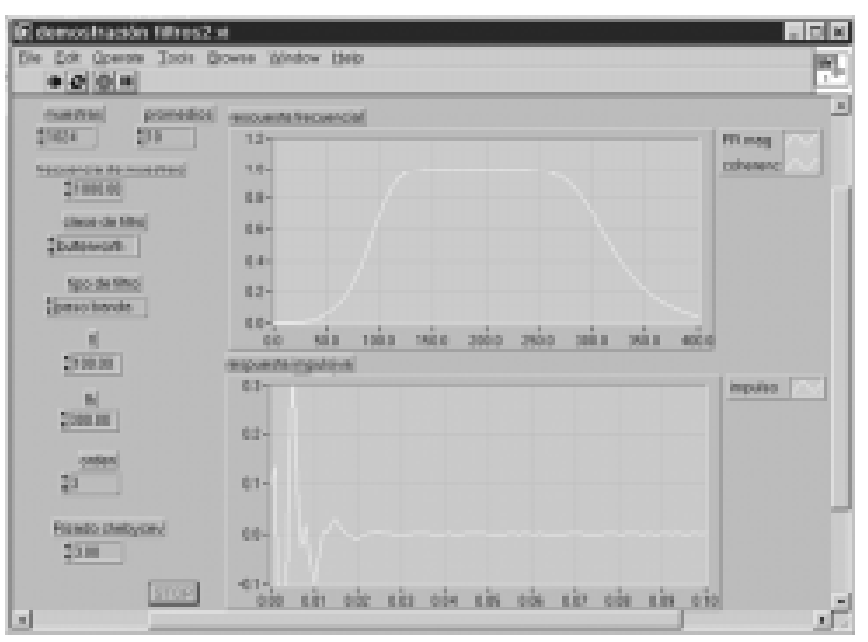

Serie exponencial de Fourier[4]:

$$
\begin{gathered}
f(t)=\sum_{n=-\infty}^{\infty} F_{n} e^{j n w_{0} t} \\
t_{1}<t<t_{2} \\
F_{n}=\frac{1}{t_{2}-t_{1}} \int_{t_{1}}^{t_{2}} f(t) e^{-j n w_{0} t} d t
\end{gathered}
$$

Serie de Fourier trigonométrica[4]:

$$
\begin{gathered}
f(t)=a_{o}+\sum_{n=1}^{\infty} a_{n} \cos n w_{o} t+\sum_{n=1}^{\infty} b_{n} \operatorname{senn} w_{o} t \\
f(t)=\sum_{n=0}^{\infty} c_{n} \cos \left(n w_{o} t+\phi_{o}\right) \\
a_{o}=\frac{1}{t_{2}-t_{1}} \int_{t_{1}}^{t_{2}} f(t) d t \\
a_{n}=\frac{2}{t_{2}-t_{1}} \int_{t_{1}}^{t_{2}} f(t) \cos n w_{o} t d t \\
b_{n}=\frac{2}{t_{2}-t_{1}} \int_{t_{1}}^{t_{2}} f(t) \operatorname{senn} w_{o} t d t \\
c_{n}=\sqrt{a^{2}+b^{2}} \\
\phi_{n}=\tan ^{-1}\left(\frac{-b_{n}}{a_{n}}\right)
\end{gathered}
$$




\section{TOPICOS DE ENSEÑANZA}

Tal como se ha mencionado antes, se establece que con el instrumento virtual diseñado, se pueden visualizar diferentes tipos de formas de onda y sus respectivos espectros de frecuencia. También se observa el efecto que produce la inserción de un filtro en el procesamiento de la señal proveniente del generador mediante la visualización de la señal de salida del filtro tanto en el dominio del tiempo como en el dominio de la frecuencia.

De otro lado, también podemos ver la característica de entrada, salida (Diagrama de bode y respuesta impulsiva) de los filtros utilizados.

\section{RESULTADOS}

7.1 Filtro Butterworth para una onda triangular, pasa banda, orden 2, ver figura 14 :

Figura 14. Panel de control Filtro Butterworth.

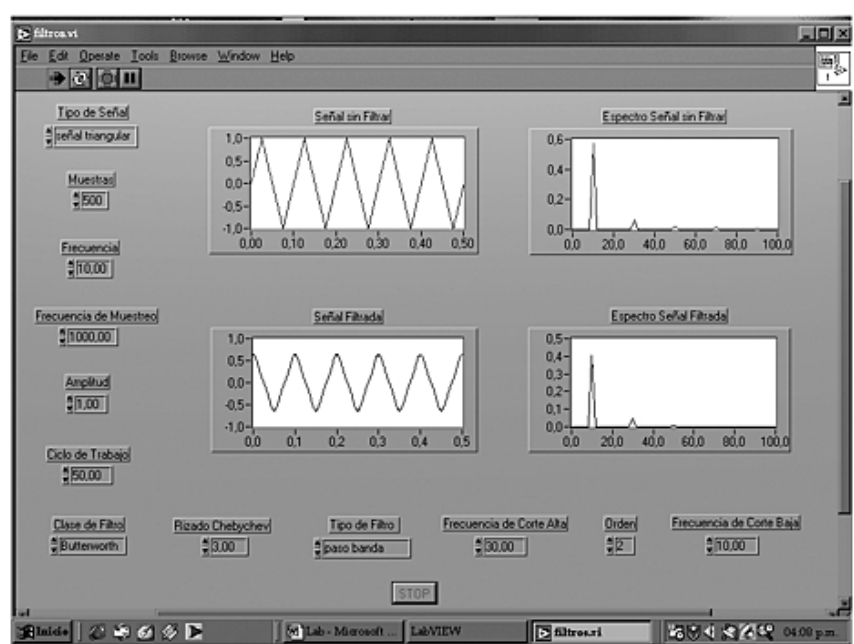

7.2 Filtro Chebychev para una onda triangular, pasa banda, orden 2, ver figura 15 :
Figura 15. Panel de control Filtro Chebychev.

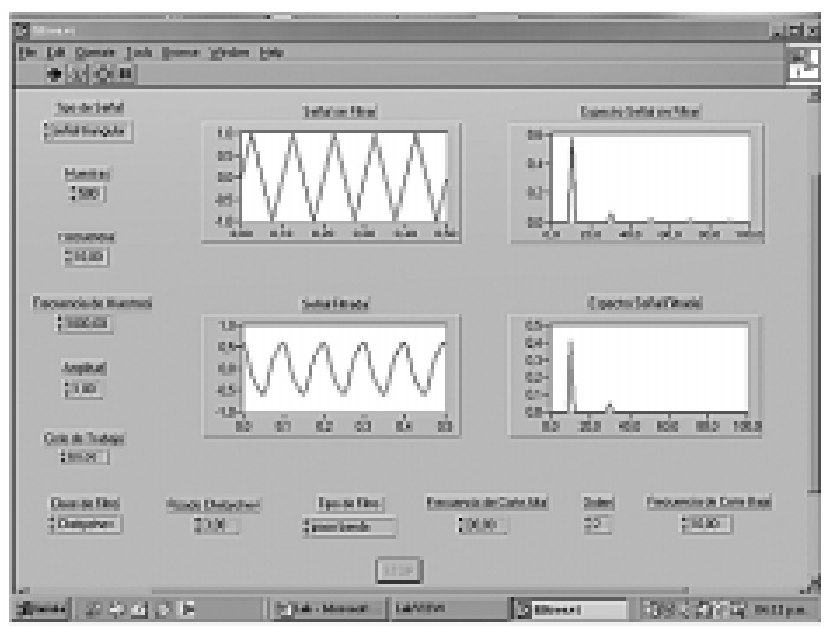

\section{CONCLUSIÓN}

- Filtro $\mathrm{VI}$ es un instrumento virtual desarrollado, utilizando el lenguaje de programación gráfico LabView que presenta las siguientes características:

1. Permite la visualización en tiempo real del efecto que produce la inserción de un filtro en el procesamiento de una señal.

2. Es tolerante a posibles errores en los datos de entrada.

3. Los profesores y estudiantes no requieren un conocimiento previo para el manejo del instrumento virtual.

- Aporta las características esenciales de los instrumentos virtuales y de la programación gráfica.

- Analizan las implicaciones didácticas que tiene la incorporación de la instrumentación virtual en la enseñanza de la ingeniería electrónica de la UFPS.

- Se presenta las diferencias de los filtros Butterworth y Chebychev. 


\section{REFERENCIAS}

[1] Rafael Chacón Rúgeles, en su articulo "la instrumentación virtual en la enseñanza de la ingeniería electrónica" UNET, San Cristóbal Republica Bolivariana de Venezuela.

[2] Manuales de labview, curso ofrecidos por la National instrument, Curso I, II. 2000 www.ni.com

[3] Neira Domínguez, Carmen de Haro, Roset Mas, Desarrollo de instrumentación virtual para biosensores amperimétricos, instituto de transductores de microelectrónica de Barcelona (IMB-CNM) campus UAB. Nerina.Domínguez@cnm.es

[4] Chia-Nan Chang, Hui-Kang Teng, Jun-Yuan Chen, and Huang-Jen Chiu, Computerized Conducted EMI Filter Design System Using LabVIEW and Its Application, Department of Electronic Engineering National Taiwan University of Science and Technology Taipei, Taiwan, R.O.C. (Received May 1, 2000; Accepted August 7, 2000).

[5] César San Martín, Flavio Torres, laboratorios virtuales. uso de internet para el trabajo colaborativo, Depto. Ing. Eléctrica - Universidad de La Frontera Casilla 54 -D Temuco Email: csmarti@ufro.cl

Recibido: Agosto 20 de 2004

Aceptado: Junio 16 de 2005 